\title{
Edge-Fog Cloud: A Distributed Cloud for Internet of Things Computations
}

\author{
Nitinder Mohan, Jussi Kangasharju \\ Department of Computer Science, University of Helsinki, Finland \\ Email: \{firstname.lastname@cs.helsinki.fi\}
}

\begin{abstract}
Internet of Things typically involves a significant number of smart sensors sensing information from the environment and sharing it to a cloud service for processing. Various architectural abstractions, such as Fog and Edge computing, have been proposed to localize some of the processing near the sensors and away from the central cloud servers. In this paper, we propose Edge-Fog Cloud which distributes task processing on the participating cloud resources in the network. We develop the Least Processing Cost First (LPCF) method for assigning the processing tasks to nodes which provide the optimal processing time and near optimal networking costs. We evaluate LPCF in a variety of scenarios and demonstrate its effectiveness in finding the processing task assignments.
\end{abstract}

Keywords-Cloud Computing, Fog Computing, Edge Computing, Internet of Things, Task Assignment

\section{INTRODUCTION}

Internet of Things (IoT) typically involves a large number of smart sensors sensing information from the environment and sharing it to a cloud service for processing. A recent study by National Cable \& Telecommunications Association (NCTA) assumes that close to 50.1 billion IoT devices will be connected to the Internet by 2020 [1]. This leads to two major issues for computing IoT-generated data: i) the processing time of time-critical IoT applications can be limited by the network delay for offloading data to cloud, and ii) uploading data from a large number of IoT generators may induce network congestion thus incurring further network delay.

To tackle network issues involved in IoT and similar application's computation, researchers have proposed bringing the compute cloud closer to data generators and consumers. One proposal is Fog computing cloud [2] which lets network devices run cloud application logic on their native architecture. The objective of Fog cloud is to perform low-latency computation/aggregation on the data while routing it to the central cloud for heavy computations [3], [4]. On the other hand, Edge-centric computing cloud [7] takes inspiration from projects such as SETI@Home, Folding@Home etc. [5], [6], and proposes a consolidation of human-operated, voluntary resources such as desktop PCs, tablets, smart phones, nano data centers as a cloud. As the resources in Edge cloud usually lie in one-hop proximity 978-1-5090-4960-8/16/\$31.00 (C)2016 IEEE to the IoT sensors; processing the data at the edge can significantly reduce the network delay [8], [9].

While both Edge and Fog cloud envision bringing the cloud closer to the users, the two approaches only utilize their resources to carry out pre-processing tasks thus relying on a centralized cloud for heavy, computationally intensive tasks. This semi-dependence on a central cloud works well for applications which require tight data and compute coupling but proves disadvantageous for applications which generate large amounts of distributed data interactive user involvement.

In this paper, we present a node-oriented, fully decentralized hybrid of Edge and Fog compute cloud model, Edge-Fog cloud. As the name suggests, the outermost layer of the Edge-Fog cloud is composed of a large number of volunteer, human-operated edge devices connected via adhoc network chains. The inner layer is composed of a dense network of Fog devices with high compute capabilities. Due to its decentralized architecture, the Edge-Fog cloud is capable of decoupling processing time from network delays by effectively handling processing close to the data generators. Edge-Fog cloud offers reliable data storage of raw and computed data at the central data store located at the core of its architecture.

The contributions we make in this paper are as follows:

- We present Edge-Fog cloud architecture, which is based on classifying compute devices into Edge and Fog layers, depending on their capabilities and ownership.

- We design LPCF algorithm which assigns tasks on the available nodes in the Edge-Fog cloud while minimizing the processing time and network costs. We show that LPCF achieves near-optimal networking costs in polynomial time as opposed to exponential time complexity.

- We develop an Edge-Fog cloud simulator and integrate it with LPCF assignment solver. We demonstrate and compare the efficiency of LPCF with its related works across a range of parameters and simulations.

- We discuss and provide insights regarding the characteristics of Edge-Fog cloud that will affect its performance in real-world.

The remainder of the paper is organized as follows. In 


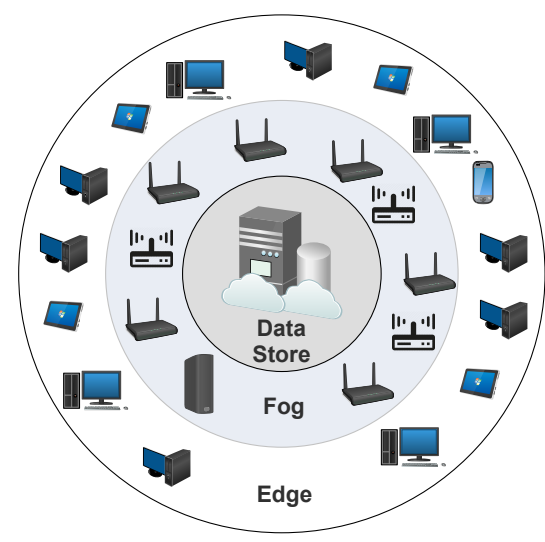

Figure 1: Proposed Edge-Fog cloud architecture

Section II, we describe the Edge-Fog cloud architecture. We propose our approach for deploying compute application tasks on the Edge-Fog cloud in efficient manner in Section III. In Section IV and $\mathrm{V}$ we evaluate the effectiveness of our LPCF algorithm and discuss EdgeFog deployment issues. We discuss the related work in Section VI. Section VII concludes the paper.

\section{Edge-Fog Cloud}

\section{A. Architecture}

Figure 1 shows the architecture of the Edge-Fog cloud. Unlike the network-oriented view of the traditional cloud model, the Edge-Fog cloud takes a node-oriented approach wherein the model is divided into three layers comprising of different resource types.

Edge Layer. The outermost layer of the cloud is Edge layer. The Edge is a collection of loosely coupled, voluntary $^{1}$ and human-operated resources such as desktops, laptops, nano data centers, tablets, etc. As the name suggests, the resources reside at the edge of the network and are within one/two-hop distance from the IoT sensors and clients. Edge resources have varying ranges of computational capabilities from highly capable devices such as workstations, nano data centers etc. to less capable such as tablets or smart phones. Edge layer resources are assumed to have device-to-device connectivity within the layer and reliable connectivity to Fog layer.

Fog Layer. The Fog layer resides on top of the edge and is a consolidation of networking devices such as routers and switches with high computing capabilities and ability to run cloud application logic on their native architecture. We envision Fog resources to be manufactured, managed and deployed by cloud vendors (such as CISCO [10]). As Fog layer forms the network backbone of Edge-Fog cloud, the resources in this layer are interconnected with highspeed, reliable links. Moreover, Fog resources reside farther from the edge of the network when compared to Edge layer

\footnotetext{
${ }^{1}$ Several incentive/credit mechanisms can be employed for devices to volunteer as Edge resource. However, discussion of such mechanisms is currently out-of-scope of this paper.
}

but closer than a central cloud. Fog is used to effectively handle computationally intensive tasks offloaded by Edge resources.

Data Store. Unlike the traditional cloud model, the core of the Edge-Fog cloud has no computational capabilities and only serves as a repository for archiving all data in the cloud. A centralized store provides reliability and easy access to data by any computing resources in the cloud. Being at the core of the architecture, the Data Store is accessible by both Edge and Fog layers.

\section{B. Benefits of the Edge-Fog cloud}

The Edge-Fog cloud offer several benefits:

1) Reduced network load: The Edge-Fog cloud provides computation at the edge of the network near the IoT generators thus reducing the amount of data that flows in the network.

2) Native support for mobility: Mobility along with reliability is a quintessential requirement for many IoT applications. Edge resources such as smartphones or laptops can offer native physical and virtual mobility for supporting such mobile IoT applications.

3) Providing context: Resources in Edge-Fog cloud also provide contextual awareness to data generated by sensors. Edge resources play a role in combining data from sensors using location or application contexts.

4) No single point of failure: As computation in EdgeFog cloud is completely decentralized, the model has no single point of failure. Several snapshots of an application can be deployed on the cloud for increased reliability.

Applications such as connected vehicles, energy monitoring, automated traffic control etc. can highly benefit from Edge-Fog cloud as most of the tasks in such applications are distributed and network-constrainted.

\section{Task Deployment on Edge-Fog Cloud}

The Edge-Fog cloud is a scalable platform for a large number of interconnected Edge and Fog devices and efficiently utilize the processing power they offer. However, as the devices in the Edge-Fog cloud are governed by certain processing and network capabilities, deploying tasks on these devices has an associated cost. A typical task deployment algorithm must map a job node from the job graph to an Edge/Fog resource. The cost of deployment is dependent on both the properties of resources and that of the deployed task itself. For example, the more coordination needed by task with its peers for completion, the higher will be the associated network cost. In order to provide a scalable and efficient solution, the task deployment algorithm for Edge-Fog cloud should find the deployment snapshot with least possible cost without unduly impacting the overall completion time of that process.

Figure 2 shows a snapshot of Edge-Fog cloud of three Edge and two Fog resources. Edge and Fog is represented 


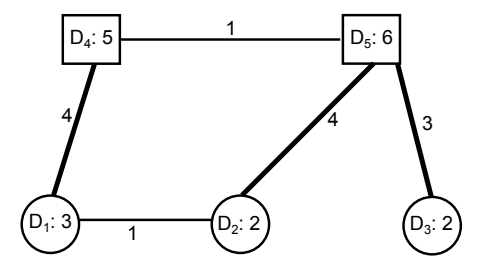

(a) Edge-Fog cloud resource graph

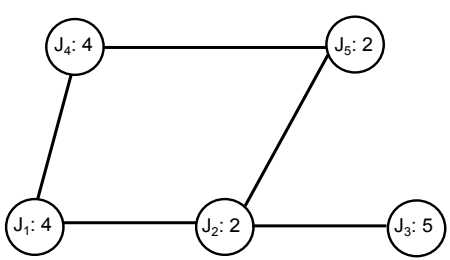

(b) Available jobs dependence graph

Figure 2: Deployment example. Each job in Figure 2(b) needs to be deployed on a resource in Figure 2(a).

by circular and rectangular nodes respectively. The link weight denotes the distance/communication cost between two devices. The processing power of each device is listed along with its label. Figure 2(b) shows the job graph to be deployed on the Edge-Fog cloud. We assume only twoway dependency between the jobs wherein Job $J_{1}$ and $J_{2}$ are dependent on each other if there exists a connection between them. The size of each job, listed along with its label, denotes the processing power required to complete the job.

We assume that the number of tasks and devices to be equal while task deployment. If there are more tasks than devices, we split existing devices into virtual devices such that their number is equal to the number of tasks. In the opposite case, we simply ignore the superfluous devices.

\section{A. Network Only Cost (NOC) Assignment}

Previous works have tried to model task deployment algorithms which minimize the associated networking cost [11]. The formal definition of such task assignment strategies is to find an assignment which places $\mathcal{N}$ jobs on $\mathcal{N}$ devices such that the associated network cost is minimized. For the rest of the paper, we refer to all such algorithms as Network-Only Cost (NOC) algorithms. Let $D_{\text {conn }}(i, j)$ represent the cost of connectivity between the devices $D_{i}$ and $D_{j}$ and $J_{\text {conn }}(i, j)$ denote the dependency between the jobs $J_{i}$ and $J_{j}$. Both $D_{c o n n}$ and $J_{c o n n}$ are square matrices of size $\mathcal{N} \times \mathcal{N} . f(i)$ signifies the constraint of assigning a particular job to a device.

With $\mathcal{N}$ devices/jobs, the search space of possible assignments in NOC is $\mathcal{N}$ !. For example, in Figure 2, the assignment $D_{1} \rightarrow J_{1} ; D_{2} \rightarrow J_{2} ; D_{3} \rightarrow J_{3} ; D_{4} \rightarrow J_{4}$; $D_{5} \rightarrow J_{5}$ has network cost 17 , whereas, the assignment $D_{1} \rightarrow J_{4} ; D_{2} \rightarrow J_{5} ; D_{3} \rightarrow J_{3} ; D_{4} \rightarrow J_{1} ; D_{5} \rightarrow J_{2}$ has cost 13. A naive NOC implementation would iteratively search for the assignment with least possible cost in the entire search space thus having the worst case complexity of $\mathrm{O}(\mathcal{N} !)$. On the other hand, NOC closely resembles the well-known Quadratic Assignment Problem (QAP) [12]. QAP generalizes minimal cost assignment as:

$$
\sum_{i, j \in A} J_{\text {conn }}(i, j) * D_{\text {conn }}(f(i), f(j))
$$

where $A$ is set of all arcs in the graph.

However, QAP is an NP-hard problem and its solution

\begin{tabular}{|l|c|c|c|c|c|c|c|}
\hline Topology size & 5 & 10 & 15 & 30 & 60 & 100 & 150 \\
\hline $\begin{array}{l}\text { Original search } \\
\text { space }\end{array}$ & $5 !$ & $10 !$ & $15 !$ & $30 !$ & $60 !$ & $100 !$ & $150 !$ \\
\hline $\begin{array}{l}\text { LPCF search } \\
\text { space }\end{array}$ & $1 !$ & $3 !$ & $>4 !$ & $>5 !$ & $>7 !$ & $>8 !$ & $>9 !$ \\
\hline
\end{tabular}

Table I: Problem search space reduction in LPCF

can only be approximated by applying constraints. Computing the optimal deployment for a problem space of 30 nodes using QAP may take up to a week on a computational grid comprising of 2500 machines [14]. Branch-andbound based algorithms such as Gilmore-Lawler Bound (GLB) or Hungarian bounds can estimate the solution for small-sized QAP problems. Since the job scheduling on an Edge-Fog cloud may encompass computing an assignment of hundreds of devices, a more efficient algorithm for finding an optimal task assignment is needed.

\section{B. Least Processing Cost First (LPCF) Assignment}

As the Edge resources of the Edge-Fog cloud may not be highly processing-capable, the task assignment algorithm must also consider the associated processing cost of deployment. We thus propose LPCF, a task assignment solver which first minimizes processing cost of the assignment and further optimizes the network cost. In section IV we show that LPCF algorithm is highly scalable when compared to NOC based algorithms. LPCF computes its optimal task assignment in the following manner.

1) Optimize the associated processing cost: LPCF calculates the processing cost associated with each possible assignment in the search space. The minimization function used by LPCF is:

$$
\sum_{i, j \in A} C\left(\frac{J_{\text {size }}(i)}{D_{\text {proc }}(j)}\right) x_{i j}
$$

where $C$ denotes the overall cost function; $J_{\text {size }}$ and $D_{\text {proc }}$ are matrices of size $1 \mathrm{x} \mathcal{N}$ representing the job sizes and the processing power of involved devices respectively. $x_{i j}$ is a binary job assignment variable.

Eq. 2 is an objective function of Linear Assignment Problem (LAP) which unlike QAP, is polynomial [15]. Algorithms such as Kuhn-Munkres/Hungarian guarantee an optimal solution for this problem in $O\left(n^{3}\right)$ (worst case). The first step of LPCF employs such an algorithm to compute an assignment which has the least associated processing cost. 
2) Reducing the sub-problem space size: As the Edge-Fog cloud consists of several homogeneous devices with similar processing capabilities, interchanging jobs assigned on any such two devices does not alter the associated processing cost. The same argument is also applicable to homogeneous jobs in job graph. To illustrate, using equation 2 the assignment $D_{1} \rightarrow J_{1} ; D_{2} \rightarrow J_{2} ; D_{3} \rightarrow J_{3}$; $D_{4} \rightarrow J_{4} ; D_{5} \rightarrow J_{5}$ in Figure 2 has the processing cost of 5.97 which remains the same if we interchange the jobs deployed on $D_{1}$ and $D_{4}$.

LPCF computes all possible compositions of the assignment computed in the first step and forms a smaller search space of assignments with least associated processing cost. Table I shows the reduction in problem search space achieved by LPCF.

3) Accounting network cost of the assignment: In this step, LPCF computes the network cost associated with each assignment in the reduced problem search space and chooses the one with least network cost. Note that as the optimal assignment is updated at each iteration of the exhaustive search of sub-search space, a branch-andbound variant of the algorithm can find the assignment within a time bound for large search space sizes. Thus, the assignment computed by LPCF has the least associated processing cost and almost optimal network cost.

Our approach has several advantages over NOC-based algorithms. The most fundamental of them is that unlike the NOC assignment, our algorithm guarantees an assignment in polynomial time thus significantly reducing the deployment calculation time. Moreover, as not all devices in the Edge-Fog cloud are highly processing capable, LPCF also takes into account the processing cost of the assignment.

\section{Evaluation}

We now evaluate the computation complexity for deploying jobs on several different Edge-Fog topologies. We have designed and implemented an Edge-Fog cloud simulator in Python (simulator code is available at [13]). The simulator generates a network of Edge and Fog resources and a job dependence graph based on several user-defined parameters. Table II shows the default parameter values we use for evaluating Edge-Fog cloud in this paper.

We further implement and integrate LPCF task assignment solver in the Edge-Fog cloud simulator. To compare, we measure the performance of LPCF against two variants of NOC task assignment solver, permutation-based and QAP-based. For the QAP-based variant of NOC, we use an open-source implementation of Kuhn-Munkres solver available from QAPLIB [12].

\section{A. Processing time analysis}

We analyze the overall processing time for computing an assignment by LPCF and NOC algorithms for several problem sizes. We set the maximum completion time of computation to one hour. The results are in Table III.

\begin{tabular}{|l|l|}
\hline Properties & Value \\
\hline Total number of devices/jobs & Experiment \\
Number of Edge devices & specific \\
Number of Fog devices & $40 \%$ of total \\
Processing power of Edge resources & $2-5$ \\
Processing power of Fog resources & $7-9$ \\
Connection density in Edge layer (0-1) & 0.2 \\
Connection density in Fog layer (0-1) & 0.6 \\
Connection density between & 0.5 \\
Edge and Fog layer (0-1) & 2 \\
Lowest job size in job pool & 6 \\
Highest job size in job pool & 0.2 \\
Inter-dependence density between jobs (0-1) & \\
\hline
\end{tabular}

Table II: Default parameter values of Edge-Fog cloud simulator

It is evident from the results that LPCF performs much better than both NOC-based solvers. For 30 node topology, where both solvers are unable to find an optimal assignment within the time limit, LPCF computes its assignment in under a second. For large topologies of 150 nodes, LPCF exceeds the maximum allotted time for the computing an optimal assignment. The primary reason for this increased computation time is due to the large size of the reduced search space size in LPCF. The current implementation of LPCF iteratively searches for the optimal assignment in reduced problem space which can be costly. However, a branch-and-bound variant of LPCF can significantly reduce the search time thus reducing the overall computation time.

\section{B. Comparative study of associated costs}

Figure 3(a) compares the cost minimization achieved by LPCF when compared to NOC QAP task assignment solver. The minimum/maximum bounds are obtained by choosing the $\mathcal{N}$ smallest/largest link costs in the EdgeFog cloud resource graph. It should be noted that the minimum/maximum cost depicted might not belong to a valid assignment as it does not consider job dependencies.

It is evident from the figure that even though the assignment computed via LPCF first optimizes processing time for assignment, the associated network cost is within $10 \%$ range of the optimal value computed by the NOC. Also, we can see from Table II that the QAP-based NOC solver have significantly higher computing time when compared to LPCF. We further implemented a branch-andbound variant of QAP solver which approximates the best solution within the specified time limit. We then limit the computation time of QAP to that of LPCF and plot the associated network costs of the optimal assignments found by both these algorithms. The plot is shown in Figure 3(b). Here we see that for large topologies, the assignment computed by LPCF has lower associated network cost than that computed by NOC.

Figure 3(c) compares the associated processing cost of assignments computed by the two solvers. As unlike NOC, the assignment computed via LPCF is optimized on processing cost, the associated processing cost of the 


\begin{tabular}{|c|c|c|c|c|c|c|c|c|c|c|}
\hline $\begin{array}{l}\text { Number of } \\
\text { Devices/Jobs }=\end{array}$ & 5 & 10 & 15 & 20 & 30 & 40 & 50 & 60 & 100 & 150 \\
\hline NOC Permutation solver & $0.068 \mathrm{~s}$ & $23 \mathrm{~m} 20.785 \mathrm{~s}$ & $>1 \mathrm{~h}$ & $>1 \mathrm{~h}$ & $>1 \mathrm{~h}$ & $>1 \mathrm{~h}$ & $>1 \mathrm{~h}$ & $>1 \mathrm{~h}$ & $>1 \mathrm{~h}$ & NA \\
\hline NOC QAP solver & $0.026 \mathrm{~s}$ & $36.273 \mathrm{~s}$ & $3 \mathrm{~m} \mathrm{22.508s}$ & $18 \mathrm{~m} \mathrm{38.23 \textrm {s }}$ & $>1 \mathrm{~h}$ & $>1 \mathrm{~h}$ & $>1 \mathrm{~h}$ & $>1 \mathrm{~h}$ & $>1 \mathrm{~h}$ & NA \\
\hline $\mathrm{LPCF}$ & $0.0005 \mathrm{~s}$ & $0.002 \mathrm{~s}$ & $0.044 \mathrm{~s}$ & $0.045 \mathrm{~s}$ & $0.18 \mathrm{~s}$ & $0.82 \mathrm{~s}$ & $4.358 \mathrm{~s}$ & $26.85 \mathrm{~s}$ & $7 \mathrm{~m} 3 \mathrm{~s}$ & $>1 \mathrm{~h}$ \\
\hline
\end{tabular}

Table III: Optimal assignment computation time

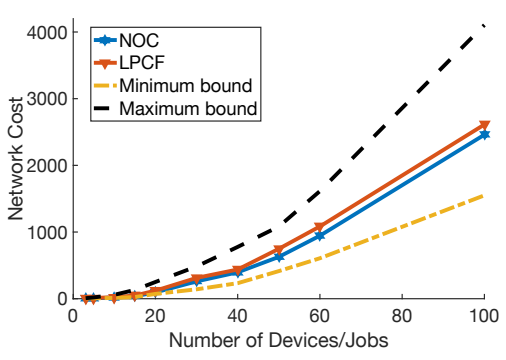

(a) Network cost comparison between LPCF and NOC

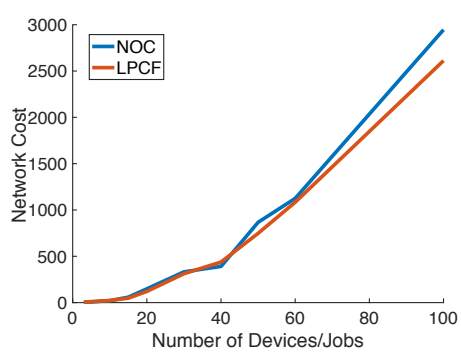

(b) Network time comparison within time limit

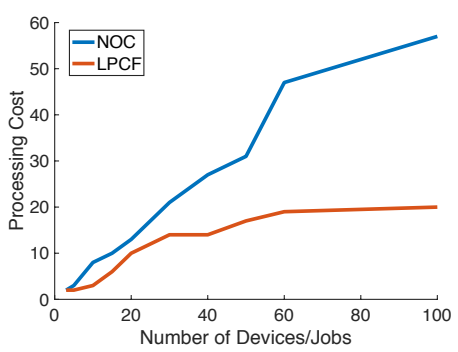

(c) Processing cost analysis

Figure 3: Edge-Fog cloud associated cost analysis

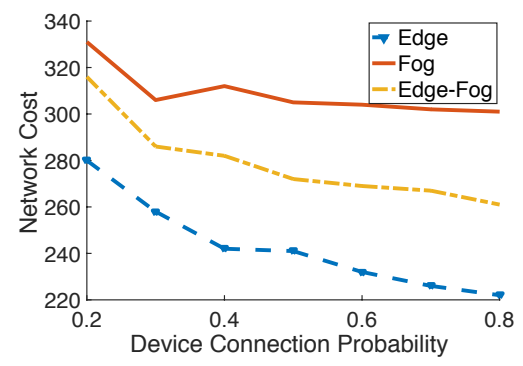

Figure 4: Network cost variation with inter-device connection densities

assignment computed by LPCF is always lower than that computed by NOC.

\section{Discussion}

Q1. Which node is responsible for running the assignment solver?

The LPCF algorithm needs a centralized controller for managing the execution of the algorithm, however, its actual execution can be distributed and is not dependent on any single node. One node needs to be able to get the snapshot of the system state (availability of nodes and costs of links) and we assume this snapshot to remain constant during the execution of the algorithm. Calculating the individual assignment permutations for processing or networking costs in steps 1 and 3 can be distributed to other nodes or can be performed by the controller. The LPCF algorithm can thus be executed by any of the nodes in the system, whether an Edge node or a Fog node. We do not consider the cost of running the algorithm in our evaluation since the overheads are similar for both LPCF and NOC QAP (namely obtaining the snapshot and going through the permutations).

Q2. How well should the devices in the Edge-Fog cloud be connected to each other?

Edge layer has optimistic connections within itself whereas the Fog layer has dense network connections; but

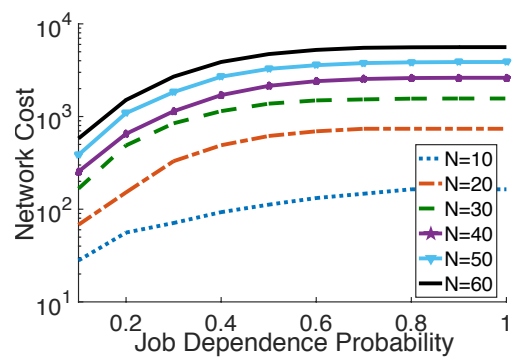

Figure 5: Effect of job dependence on network cost of assignment

the inter-layer connections between the Edge and the Fog are much higher cost and spans multiple hops. We try to find the optimal connection density of each layer (and inter-layer connections as well) such that the resulting assignment has low associated network cost. We increase the connection density of each layer from $20 \%$ to $80 \%$ and plot the changes in network cost of assignment computed by LPCF in Figure 4.

As we increase the connection density of Edge, Fog and interconnections we see a decrease of $\sim 21 \%, \sim 9 \%$ and $\sim 17 \%$ in network cost respectively. The inter-layer connections play a major part in resulting network cost; increasing their density impacts the overall cost much more. We can infer that deploying jobs in an Edge-Fog cloud which have well-connected devices in edge layer and dense connections between edge and fog layers, the overall cost of deployment is significantly reduced.

Q3. Do the properties of job graph deployed on the Edge-Fog cloud also affect the overall cost?

In Figure 5, we change the interdependence of the job graph deployed on the Edge-Fog cloud from 10\% to $100 \%$ and calculate the network cost associated with the deployment. We then deploy the job graph on several topology sizes of Edge-Fog cloud.

The results clearly show that higher dependence be- 
tween the jobs result in a higher network cost. This is because the dependence links between the sub-jobs are mapped to the links between the devices of the Edge-Fog. Larger dependence links map to a mesh of device linkages thus leading to an increased network cost. It can also be seen from the figure that after a particular job dependency value, the associated network cost of assignment stabilizes. This is primarily because after a particular job interdependence all heavy links of the device graph are part of the computed assignment and adding more links does not change the overall network cost significantly.

\section{RELATED WORK}

Cloudlets [16] propose a small-scale, localized cloud installed at the edge of the network along with the centralized cloud and is based on virtualization technologies. Several other works have explored combining stable peerresources as nano data centers, micro clouds, community clouds, etc., for compute/storage tasks [17]-[20].

Several researchers have proposed to bring part of the cloud closer to the edge of the network. Following the Fog cloud characteristics proposed by CISCO [2], Bonomi et al. [21] and Yannuzzi et al. [3] show that the fog is the appropriate platform for loosely coupled, computationally intensive IoT-based applications, such as connected vehicles and smart cities. Hong et al. [4] provide a programming model and API for developing applications on the Fog cloud. On the other hand, unlike installing managed compute resources as Fog devices to process cloud applications, Lopez et al. [7] propose a semi-centralized cloud architecture, Edge cloud, composing of volunteerbased, user-centric compute resources. Likewise, Ryden et al. [22] proposed a dispersed cloud, Nebula, which utilizes volunteer resources for running data-intensive tasks. The authors discuss the effectiveness of their approach by deploying Map-reduce jobs on available resources.

Our work differs from all these approaches as unlike them, wherein a central entity schedules and processes several application tasks; Edge-Fog cloud proposes an entirely decentralized computing mechanism. Due to its unique nodular and layered architecture, the EdgeFog cloud natively supports computations on distributed, semi-dependent data produced by IoT.

\section{Conclusion}

In this paper, we proposed the Edge-Fog cloud, a decentralized cloud model for handling computation-based, high volume and distributable data such as that generated by IoT. The model builds on the existing Edge and Fog cloud approaches and provides data resilience through a centralized data store. We also provided a novel task allocation mechanism for Edge-Fog cloud which significantly reduces the deployment time without sacrificing the associated cost when compared to related approaches. Further, we address several questions which might impact the realworld implementation of Edge-Fog cloud.
Future work in the area includes considering practical implementation and deployment issues of LPCF in a realistic Edge-Fog scenario.

\section{ACKNOWLEDGEMENT}

This research was funded by the joint EU FP7 Marie Curie Actions Cleansky Project, Contract No. 607584.

\section{REFERENCES}

[1] Broadband by the numbers,'https://www.ncta.com/broadbandby-the-numbers', accessed: 2015-04-22.

[2] CISCO, "Cisco fog computing solutions: Unleash the power of the internet of things (whitepaper)," 2015. [Online].

[3] M. Yannuzzi et al. "Key ingredients in an iot recipe: Fog computing, cloud computing, and more fog computing," in IEEE CAMAD, 2014.

[4] K. Hong et al. "Mobile fog: A programming model for large-scale applications on the internet of things," in $A C M$ SIGCOMM Workshop on Mobile Cloud Computing,2013.

[5] D. P. Anderson et al. "Seti@home: An experiment in publicresource computing," Commun. ACM, vol. 45, no. 11, pp. $56-61$, Nov. 2002.

[6] A. L. Beberg et al. "Folding@home: Lessons from eight years of volunteer distributed computing," in IEEE IPDPS, 2009.

[7] P. Garcia Lopez et al. "Edge-centric computing: Vision and challenges," SIGCOMM Comput. Commun. Rev., vol. 45, no. 5 , pp. 37-42, Sep. 2015

[8] A. Chandra, J. Weissman, and B. Heintz, "Decentralized edge clouds," Internet Computing, IEEE, vol. 17, no. 5, pp. 70-73, Sept 2013.

[9] S. Islam and J.-C. Grégoire, "Giving users an edge: A flexible cloud model and its application for multimedia," Future Generation Computer Systems, vol. 28, no. 6, pp. $823-832$, 2012.

[10] CISCO, "Fog computing and the internet of things: Extend the cloud to where the things are (whitepaper)," 2015. [Online].

[11] E. Shakshuki, A. M. Haubenwaller, and K. Vandikas, "Computations on the edge in the internet of things," Procedia Computer Science, vol. 52, pp. 29 - 34, 2015.

[12] A quadratic assignment problem library, http://anjos.mgi.polymtl.ca/qaplib/, accessed: 2010-09-30.

[13] Edge-Fog simulator and LPCF solver, https://github.com/nitinder-mohan/EdgeFogSimulator.

[14] M. C. Yurko, "A parallel computational framework for solving quadratic assignment problems exactly," 2010.

[15] S. Martello, M. Minoux, C. Ribeiro, and G. Laporte, Surveys in combinatorial optimization. Elsevier, 2011, vol. 31.

[16] T. Verbelen et al. "Cloudlets: Bringing the cloud to the mobile user," in ACM Workshop on Mobile Cloud Computing and Services, 2012.

[17] I. P. Kurniawan, H. Febiansyah, and J. B. Kwon, "Cost-effective content delivery networks using clouds and nano data centers," in Ubiquitous Information Technologies and Applications. Springer, 2014, pp. 417-424.

[18] C. Shi et al. "Serendipity: Enabling remote computing among intermittently connected mobile devices," in ACM MobiHoc, 2012.

[19] Y. Li and W. Wang, "Can mobile cloudlets support mobile applications?" in IEEE INFOCOM 2014 - IEEE Conference on Computer Communications, April 2014, pp. 1060-1068.

[20] A. Mtibaa, K. A. Harras, and A. Fahim, "Towards computational offloading in mobile device clouds," in 2013 IEEE 5th International Conference on Cloud Computing Technology and Science, vol. 1, Dec 2013, pp. 331-338.

[21] F. Bonomi et al."Fog computing: A platform for internet of things and analytics," in Big Data and Internet of Things: A Roadmap for Smart Environments. Springer, 2014, pp. 169186.

[22] T. Anderson et al. "A brief overview of the nebula future internet architecture," SIGCOMM Comput. Commun. Rev., vol. 44, no. 3, pp. 81-86, Jul. 2014. 